\title{
The Application of Unconventional Design Strategy in Mechanical \\ Creative Design
}

\author{
Hong YANG \\ Wuhan Railway Vocational College of Technology, 430205, Wuhan, China \\ email: yanghong250250@163.com \\ Contact Phone Number: 13018053653
}

Keywords: Unconventional Design Strategy, Mechanical Creative Design, Converse Thinking, Bizarre Thinking

\begin{abstract}
The article is about the application of unconventional design strategy in mechanical creative design. For one thing, the basic contents of routine design and creative design are introduced. And, the unconventional design strategy is classified as two basic types after unconventional design strategy, converse thinking and bizarre thinking are explained. Then, the basic types of unconventional design strategies are discussed by applicable study with two examples based on the mechanical creative design. In the two examples, one belongs to the mechanical creative design based on the converse thinking, another belongs to the mechanical creative design based on bizarre thinking. Finally, the author points out that the research of this paper not only enriches the theory of mechanical creative design, but also offers the essential thinking ways for the designer to carry out mechanical creative design.
\end{abstract}

\section{Introduction}

There are different levels of mechanical design. Take one similar machine as a model, then, by keeping the main structure unchanged but changing some of the dimensions or sizes of the machine or replacing some parts with new ones, you can carry out design quickly. Such a design is called routine design. If you design a totally new machine or apply a new working principle in a machine, you have to create a new structure, not just imitate the existing one. This is creative design. Of course, creative design is more difficult than the routine one. Creative designs play an important part in the technical upgrading of devices and the research and development of new products. At the moment, the mechanical industry is one of the major industries of many countries. The mechanical creative design ought to be a highly valued in order to promote the development of the mechanical industry. In fact, there are often a lot of complex problems to be solved in the entire process of mechanical creative design, and the choice of mechanical creative design strategy is usually one of them. So-called mechanical creative design strategy can be simply defined as "a design idea or plan for attaining a particular goal of mechanical creative design". As you know, various unconventional design strategies are utilized in mechanical creative design, such as inverse-form strategy. So-called unconventional design strategy is a kind of design idea or plan which is built on converse thinking or bizarre thinking. The converse thinking and the usual thinking are diametrically opposed; in mechanical creative design, the converse thinking can be thought of as a kind of mainstream thinking because it is far from rare and is used frequently. The bizarre thinking which is neither the usual thinking nor the converse thinking is a 
non-mainstream thinking based on a very unique thinking angle, thinking vision, thinking model or thinking form etc; in mechanical creative design, the bizarre thinking can be thought of as a kind of non-mainstream thinking because it is rare and is used infrequently. By the definition of unconventional design strategy, the mechanical creative design based on unconventional design strategies can be divided into two basic types; the mechanical creative design based on the converse thinking and the mechanical creative design based on bizarre thinking. How does the unconventional design strategy apply in mechanical creative design? This problem will be solved from the research on two examples of mechanical creative design based on unconventional design strategy.

\section{Two Examples of the Mechanical Creative Design Based on Unconventional Design Strategy}

From a certain angle, the mechanical creative design is essentially a decision-making process. Actually, the decision-making is a matter of cardinal significance; a good decision leads to a good design, and vice versa. There are two examples of the mechanical creative design based on unconventional design strategy; one belongs to the mechanical creative design based on converse thinking, another belongs to the mechanical creative design based on bizarre thinking. Let's start from the example of the mechanical creative design based on converse thinking. As you kwon, the converse thinking way and the usual thinking way are diametrically opposed. In fact, you can often get twice the result with half the effort if you can use correct converse thinking in processes of the mechanical creative design. A cylindrical grinder is shown in Fig. 1. Its main components are bed, work table, headstock, wheel supporting frame, manual panel, tailstock, hand wheel box, etc. And, a grinding spindle which is one of the critical components of the grinding machine is built into the wheel supporting frame of it. In general, the better thermal stability of grinding machines, the higher precision and surface finish of work-pieces there will be in grinding processes, because the thermal deformation of them can have a huge impact on precision and surface finish of work-pieces in grinding processes. To solve the problem of adverse influence on precision and surface finish of work-pieces resulting from the thermal deformation of the grinding spindle, many creative design strategies are proposed. A creative design strategy based on non-constraint structure was proposed by Yamamoto and Otsuka of Tokyo Institute of Technology in the 1980s such as shown in Fig.2. In this creative design strategy for the grinding spindle, both the grinding wheel and the driven pulley are mounted on the front and rear taper seats at both ends of the main spindle without having any other constraints, respectively, and then the thermal elongation of the spindle cannot affect those of the grinding wheel and driven pulley. Obviously, the converse thinking way that the constraint structure is turned into the non-constraint structure is adopted in the example. In fact, this is a classic example of the mechanical creative design based on unconventional design strategy, and it belongs to the mechanical creative design based on the converse thinking. 


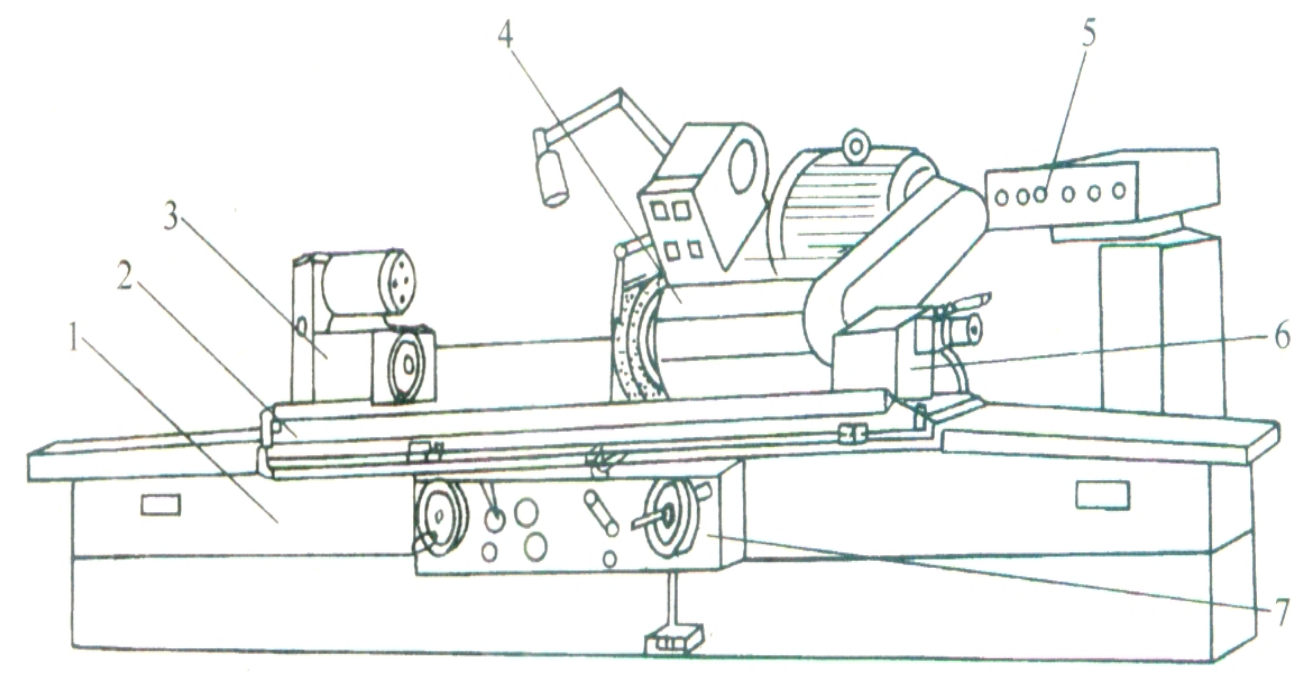

Fig. 1 A cylindrical grinder

1-Bed 2-Work table 3-Headstock 4-Wheel supporting frame

5-Manual panel 6-Tailstock 7-Hand wheel box

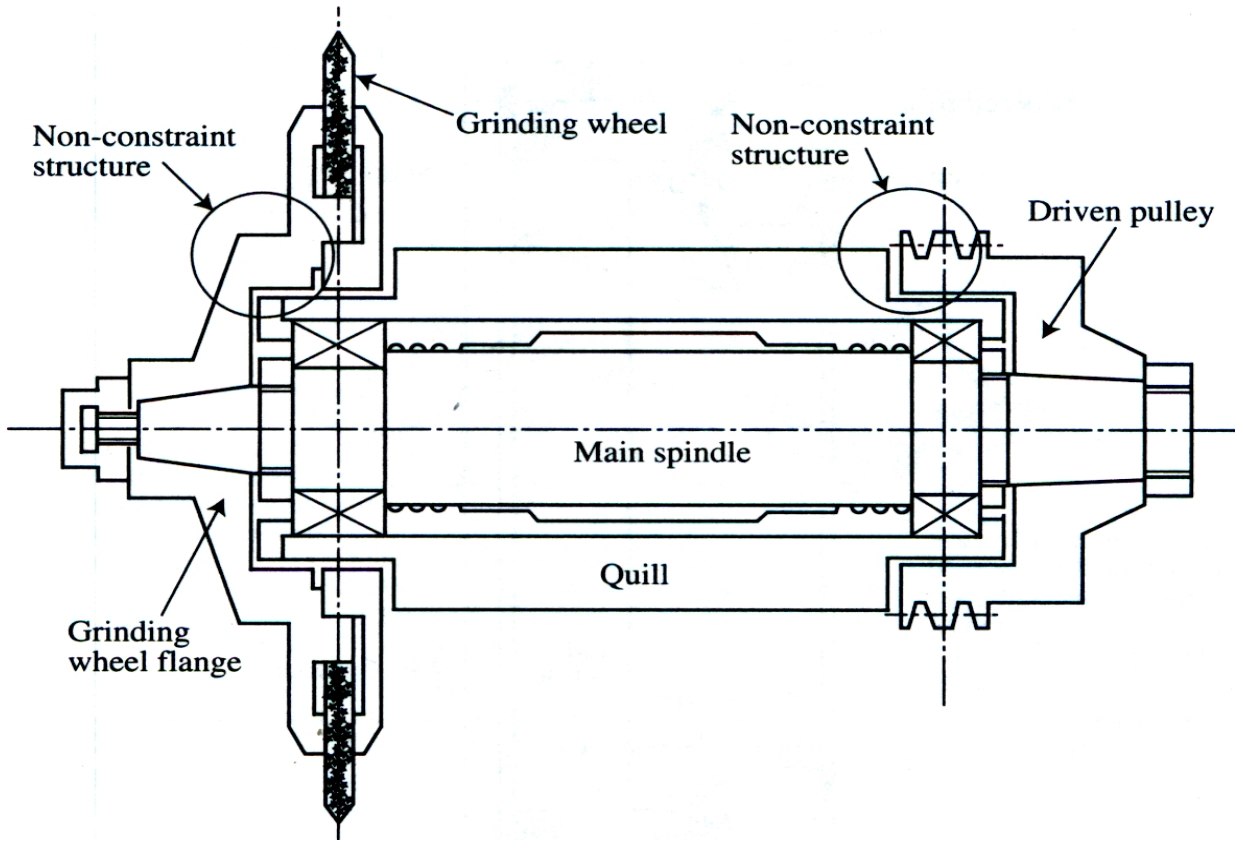

Fig. 2 Non-constraint structure in grinding spindle

Now, consider an example of mechanical creative design based on bizarre thinking. Please note: the following case study is about the design modes of the personal safety precaution for chuck wrench of the plain lathe. It's well known that conventional design modes for chuck wrench with safety precaution are shown in Fig.3 and Fig.4. Fig.3 shows a case where the design mode for chuck wrench with safety precaution is mainly based on mechanical structure. When main pole is pressed downward, the ends of main pole will be inserted in locking hole of the chuck. When main pole isn't pressed downward, the ends of main pole inserted in locking hole of the chuck will be drag out from the locking hole by elasticity of the spring in sleeve. And thus operators couldn't be hurt by the chuck wrench when the plain lathe runs. A design mode for chuck wrench with safety precaution based on the electrical control system is shown in Fig.4. When the chuck wrench is not 
inserted in the sleeve, the power switch is disconnected so that the lathe cannot run. When the chuck wrench is inserted in the sleeve, the power switch is connected and then the lathe can run. A new design mode for chuck wrench with safety precaution is shown in Fig.5. In fact, this design mode is a kind of personal safety design based on material properties. Soft tubes have a long and a short type, and a suitable type would be used as necessary. The soft tubes inserted by a cross bar is red, and the flexible elastomer bellows inserted by the main pole is black and yellow. The chuck wrench made of such colors is very attractive so that operators can draw out the chuck wrench in time. The flexible elastomer bellows of main pole is made into a compressible component. The ends of main pole inserted in locking hole of the chuck will be pulled out by the elasticity of main pole when there isn't any force that thrust the ends of main pole. And, the flexible elastomer bellows and soft tubes made from some materials having lower hardness have an enough buffing effect for the operators hit by a chuck wrench when the ends of main pole inserted in locking hole of a chuck will be pulled out because of some reasons. Therefore, the operators will not be hurt, or will be greatly done. Obviously, the design of Fig.5 is entirely based on material property with fault-tolerance. So-called fault-tolerance is that accidents and faults do not cause personal injury, or only cause slight personal injury when there are technical failures and human errors. For the design strategy, it differs from the conventional design shown in Fig. 3 and Fig.4. For the thinking way, it is neither the usual thinking nor the converse thinking. Actually, it is a kind of the mechanical creative design based on the bizarre thinking.

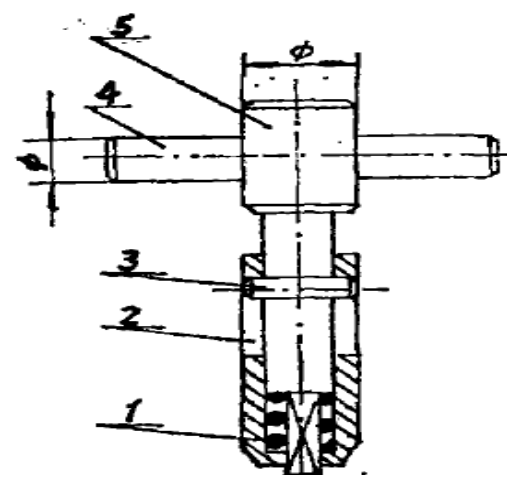

Fig. 3 A design mode for chuck wrench with safety precaution based on mechanical structure

1-spring 2-sleeve 3-pin

4-cross bar 5-main pole

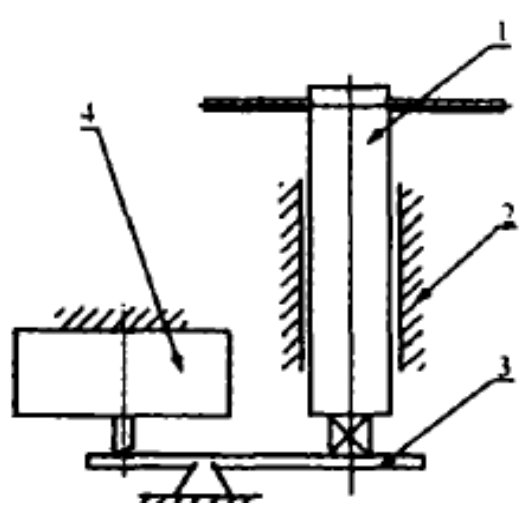

Fig. 4 A design mode for chuck wrench with safety precaution based on the electrical control system 1-chuck wrench 2-sleeve 3- lever 4- power switch 


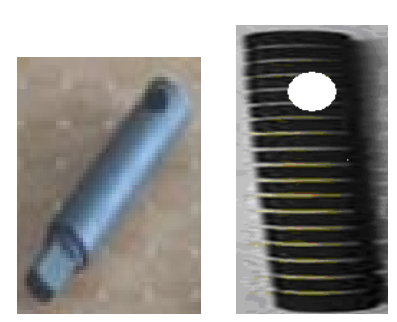

a) main pole and its flexible elastomer bellows

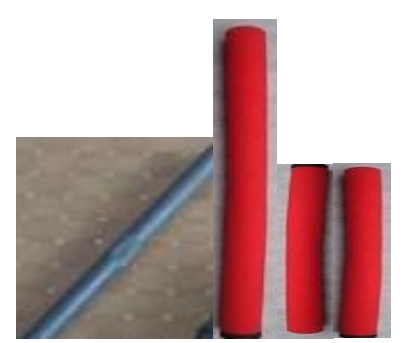

b) cross bar and its soft tubes

Fig. 5 A innovative design mode for chuck wrench with safety precaution based on mechanical property

\section{Conclusion}

Simply put, the mechanical creative design is to formulate a plan to satisfy a certain need and to create something with a physical reality. To some extent, this means that the choice of proper design strategy is essential to give a reasonable and fine plan of mechanical creative design. In fact, the analysis and determination of mechanical creative design strategy are usually one of the essential questions in the entire process of mechanical creative design. By theoretical analysis and applicable study with two examples based on unconventional design strategy, the two basic methods of mechanical creative design based on unconventional design strategies are discussed in the paper. Obviously, this is good for the theory and designer of mechanical creative design. That is, the research of this paper not only enriches the theory of mechanical creative design, but also offers the essential thinking ways for the designer to carry out mechanical creative design. Actually, there are many factors to be considered while achieving a task of mechanical creative design. In other word, a proper strategy is generally not enough to ensure that a task of the mechanical creative design is achieved successfully. Only when the designer considers in all respects of a task of mechanical creative design and has a mature plan would it be achieved successfully. The mechanical industry is one of the major industries of many countries for a long time. As the market competition is cruel day by day, the mechanical creative design becomes more important for the majority of enterprises to win and keep their competitive advantage in the furious competitive environment at present. Therefore, the study of the mechanical creative design theory and the mechanical creative design itself ought to be a highly valued in order to promote the development of the mechanical industry.

\section{References}

[1] B. S. Dhillon: Safety and Human Error in Engineering Systems (CRC Publications, New York 2012).

[2] William F. Smith and Javad Hashemi: Foundations of Materials Science and Engineering (China Machine Publications, Beijing 2011).

[3] Yoshimi Ito: Thermal Deformation in Machine Tools (McGraw Hill Publications, New York 2010).

[4] David G. Ullman: The Mechanical Design Process (China Machine Publications, Beijing 2010). 
[5] REN Weihong, XU Baoyu and PAN Zhiyon: submitted to Journal of Development \& Innovation of Machinery \& Electrical Products (2009)

[6] ZHU Changsheng, BI Xiaoyi and ZHANG Kai: submitted to Journal of Machinery Design \& Manufacture (2007)

[7] Jin Wang and Tom Ruxton: submitted to Journal of Professional Safety (1997)

[8] Varma V: submitted to Journal of Power Engineering (1996) 